\title{
Evaluation of Students Performance based on Formative Assessment using Data Mining
}

\author{
Mohak Hasmukh Gogri \\ Computer Science student \\ Fr. C.R.C.E \\ Mumbai
}

\author{
Shahla Ashraf Shaikh \\ Computer Science student \\ Fr. C.R.C.E \\ Mumbai
}

\author{
Vibha Venugopal lyengar \\ Computer Science student \\ Fr. C.R.C.E \\ Mumbai
}

\begin{abstract}
This paper proposes a system for performance assessment of students and explores how formative and summative assessment can be used to assess the performance. Proposed system focuses on formative assessment during the learning process. Computer based interactive Multiple choice question exam is proposed for formative assessment and the data generated is used for data mining. Paper discusses about the importance of detailed and instant feedback in learning process and the reduced workload on instructor in the proposed system.
\end{abstract}

\section{Keywords}

Formative assessment, summative assessment, instant feedback, interactive platform, multiple choice question (MCQ), data mining

\section{INTRODUCTION}

Assessment is one of the important components of teaching and learning cycle. The arrival of the Internet and related technologies has made online assessment systems feasible and popular in education and training. Assessment can be either formative or summative. The formative assessments are done during instruction to provide feedback to the instructors about the students' progression which allow them to respond to ongoing learning needs by modifying their instructional approaches. Formative assessments also provide immediate feedbacks to students which can enhance their learning. While the summative assessment is a test which usually given at the end of a unit, the end of a course, or the end of the year, in order to summarizes student learning and the effectiveness of the instructional program. The primary goal is to choose a method which most effectively assesses the objectives of the unit of study and helps the student to develop himself in those areas [8]. Apart from the exams, a healthy interaction between students and teachers further enhances the scope of organization development as a whole. The test results generate large amount of relative data that can be used to extract knowledge about students learning and a comparative performance amongst them is obtained. This is done by mining the data using various data mining techniques[9].

\section{LITERATURE SURVEY}

\subsection{Understanding the Impact of} Formative Assessment Strategies on First Year University Students' Conceptual Understanding of Chemical Concepts Mehmet Aydeniz and Aybuke Pabuccu[6]:

Conceptual understanding is one of the most important goals of science education. A study took place in a major university in central Turkey which used Formative Assessment as a tool to enhance student learning. In this experiment, the students had to appear for a pre-test, attend lectures and again appear for a post-test on certain topics of chemistry. The result showed that both the high and low achieving students showed a gain of $25-50 \%$ in their post-test as compared to pre-test. This proved that formative assessment strategies are effective in improving quality of student learning because teachers can identify students' misconceptions, make these misconceptions visible to the learner, and devise instructional strategies based on the feedback he/she receives from the students to address their learning needs.

\subsection{Some observations on MCQ Tests - Michael P. Coughlan[4]:}

The MCQ format is introduced to facilitate better understanding of concepts and quick self evaluation. MCQ formats used should be to speedy correction as all examiners face many questions and many student answers and deciding which answer best suits the question is ambiguous.MCQ format removes this ambiguity by defining a single answer or set of answer for a given question.

Types of MCQ

1. Single true/false/don't know type:

This are relatively easy to formulate but the $1,0,-1$ (for correct, don't know, incorrect) marking system usually used leaves a lot to be desired. The Chances of student guessing the correct answer is $50 \%$ and hence it is not desirable to test a student's knowledge on this basis.

\section{Multiple true/false/don't know type:}

In one version of this format the student is asked to state how many of 5 answers to a particular statement or question are correct. He may know that 2 are correct and 2 incorrect but get bogged down because he doesn't know whether the fifth answer is correct or not. Thus, he is penalized even though he knows $80 \%$ of the material.

\section{Matching type:}

"Match each of the examples „a" in column A with its appropriate prototype in column B". However, from the examiners' point of view there are „, $\mathrm{n}^{\text {ec }}$ answers rather than 1 to be checked. The probability of guessing the correct answer here reduces to $1 / \mathrm{n}$.

\section{Odd man Out:}

Select the option that least suits the given set of values. This method involves not only careful examination of options but analytical skills along with background knowledge. The probability of guessing the correct answer here reduces to $1 / \mathrm{n}$. 


\section{Complete the sentence:}

Normal fill in the blank(s) that checks not only for concept but also proper phrase that goes well with the given phrase.

6. One from five type:

This type (or the variant i.e. one most suitable answer type) is, rightly or wrongly, the format we have come to use most often in MCQ tests. They take the form of a statement or question followed by five possible or plausible answers. The candidate is asked to indicate the single correct (or most appropriate) answer.

\subsection{Improve Student Learning Using Online Formative Assessment System}

\section{- Shanshan Hu, Yonghua Xie[3]}

Survey questionnaire is conducted in order to collect data for analysis of research questions related to assessment methods. Results show that the key factors of an online assessment system that can effectively improve students' learning are providing instant and detailed feedback, using formative style assessment, and providing a communication tool between students and teachers.

\subsection{A case study of knowledge discovery on academic achievement, student desertion and student retention -A.Salazar, J.Gosalbez, I.Bosch, R.Miralles, L.Vergara[9]}

The large amount of data that is generated by tests can be used to enhance development of students by roping it with Data mining techniques to answer questions based on students development and factors affecting it. It can give boost to methods designed to improve students' academic performance. Knowledge management helps in summarizing information, knowledge distribution and knowledge application. A lot of research and development is in progress in the field of Data Mining to incorporate it with academics to obtain knowledge from student and give a feedback to a student, to predict a student's final marks based on his current performance, etc.

\subsection{Student Academic Performance Monitoring and Evaluation Using Data Mining Techniques-- Emmanuel N. Ogor[10]}

Performance monitoring and assessment has provided a push to help students, teachers and administrators and decision makers. The changing factors in education system has set an urge to monitor performance of students in a more efficient way by adapting data mining techniques to explore various hidden information in data by penetrating the data and investigating it to extract patterns of knowledge.

Data Mining employs Knowledge Discovery which is a process of identifying valid, new and useful patterns in data which contributes to predict outcomes by using attributes that supports decision making.

\section{FORMATIVE ASSESSMENT:}

Formative Assessment is part of the instructional process which informs both teachers and students about student understanding at a point when timely adjustments can be made. These adjustments help to ensure students achieve targeted standards-based learning goals within a set time frame. Instant feedback and reduction in teacher workload are attractive features of formative assessment.

\subsection{Instant and detailed feedback[3]:}

Feedback helps students to identify specific difficult areas and reflect on what they have learned, and what they still need to learn in order to focus learning and improve learning outcomes. Instant feedback can improve interaction between student and instructional materials. It is argued that instant feedback is time consuming for many academic staff, but the features of online assessment had lead to instant gratification for students in the form of instant feedback.

Instant feedback to learners provides elegant learning experience which can help learners to effectively analyze on what they have learned and enhance their learning outcomes. In addition, learners understand and retain information most effectively when they acquire it actively and when they receive immediate feedback. $42.4 \%$ students strongly agreed and $36.4 \%$ agreed that if feedback can be given immediately after assessment, it will be effective to validate knowledge that I understand accurately and correct misconceptions. Each stage of learning builds upon the previous stage, so the more immediate the feedback the better. If no feedback is given, then the next stage of learning may be build upon misconceptions.

\subsection{Effect on the teacher's workload[5]:}

The regular practice teachers follow is the summative format for assessing. A simple computation showed that the time required of a teacher when switching from the summative assessment to formative assessment will be increased by 5.8 times. However, one could be relieved if online technology is employed in the formative assessment. It is seen that the time required of the teacher in the formative assessment environment could be reduced from the initial 5.8 times to about 1.5 times through the use of online technology (Table 1). This reduction in teacher workload was made possible by employing online technology in the assessment exercise, instead of the teacher performing the assessment. With only a slight increase in teacher workload, the formative assessment could be practiced and hence deep learning could be promoted. Moreover, if the system is designed to record automatically the results of students' work, an alert system could also be designed into the system to alert the teacher on cases of students whose performance are not satisfactory. This also reduces the workload of the teacher to screen through the work of all the students and tracking the students' performance as they progress through their learning. 
Table 1. Minimum workload of teachers for a class of $\mathbf{4 0}$ students, considering 1 course -6 topics

\begin{tabular}{|l|l|l|l|}
\hline & & & FORMATIVE \\
& FORMATIVE & SUMMATIVE & $\begin{array}{l}\text { WITH ONLINE } \\
\text { TECHNOLOGY }\end{array}$ \\
\hline $\begin{array}{l}\text { TOTAL } \\
\text { TIME } \\
\text { TAKEN }\end{array}$ & 262 & 45 & 64 \\
\hline
\end{tabular}

Vital part of developing good MCQ based formative examination is strong database of properly classified questions. Questions need to be graded as per there difficulty level. In the initial few years of system development this will be additional load. To distribute the workload initially few institutes can collaborate and build a database together. Later on System can evolve easily with addition and modification of existing database.

\subsection{Multiple choice questions (MCQ):}

They are a very popular means of assessment and self assessment in both the traditional and electronic learning settings. They are appealing to the examinees as they can be automatically graded and they provide the capability of frequent testing, almost immediate feedback on the performance. Creation of quality questionnaire is time consuming but it is very important so as to provide credible evaluation[7]. Conclusion for the level of a question can be drawn by analyzing the overall response an individual question and thus categorizing it. A low percentage of correct answers to a particular question may indicate that the question was very difficult, or on a topic which was not covered sufficiently in teaching or simply badly drafted or misleading. A high percentage of correct answers could indicate the question is easy or contains an unintended clue to the correct response.

Some features of MCQ which prove to be helpful in assessment of students and their overall development are: [2] Objective: There must be a right answer or a best answer but there is also scope for crediting more than one right answer or 'near miss' answers, depending on the marking scheme.

Efficient: A greater part of the syllabus can be tested categorized into different difficulty levels. Such tests can be structured to include very basic questions and questions having varying levels of difficulty.

Entire marking range: MCQ scores tend to be higher than those attained in non-objective assessments, which may raise issues where the MCQs are used in summative assessment. To avoid the full marking range (0-100\%), students can be penalized for wrong answers popularly known as 'negative marking'.

Regular instant and detailed feedback: Tests made up of MCQs can be administered frequently, and thus provide regular feedback on student performance, not only to the lecturer but also to the students. Since results are quantifiable, it helps to analyze the level of student's understanding in each question which can provide detailed information about the areas he is good at and those which needs improvement.

Setting questions is time consuming and challenging: This may be a one-off expense if you recycle questions. Preparing effective feedback is also labour intensive but it is only the initial efforts.

\section{SUMMATIVE ASSESSMENT:}

Summative assessment is a test usually given at the end of semester/course, in order to summarize and grade students learning and sometimes judge the effectiveness of the instructional program. Usually essay type questions are used for summative assessment. Summative evaluation considers only the final outcomes without considering the learning process. Detailed feedback from summative assessment is of little importance. 39.4\% students strongly agree and 30.3\% agree that they have no interest in detailed feedback except they fail as they have already finished the course[3]. Also the result of summative assessment takes few weeks to come and there is less scope of detailed feedback.

To overcome the drawbacks of summative assessment techniques are developed to computerize the process. This however is out of the scope of current proposed system and can be done as future work.

\subsection{Summative assessment in Proposed System:}

Traditional essay type exams conducted are suited for summative assessment. After evaluation, the score can be uploaded in the system for Result generation.

\section{CLASSIFICATION OF STUDENTS BY DATA MINING:}

\subsection{Pre processing the data:}

The data is preprocessed before applying mining techniques. The main aim is to get rid of erroneous data by applying data cleaning, integration, transformation, reduction, discretization to fill in missing values, remove redundant data, normalize and aggregate them.

\subsection{Classification analysis:}

Using classification analysis to classify most strongly understood topic by students, a classification is done to obtain maximum secured marks by a certain number of candidates[11]. The classification technology C4.5 is used in decision tree to discover the most strongly understood subject by students. The result of this classification can coax teachers and related authorities to devote attention to particular topic. Using the threshold number of students securing marks in the topic and considering the range of marks scored as classification attribute, $\mathrm{C} 4.5$ algorithm can be used to obtain information about the topic which needs more devotion[12].

\section{PROPOSED SYSTEM:}

The system of assessment proposed collects all the positive features from the above mentioned modules. For formative assessment, system proposes a unique Interactive MCQ based exam. Here the questions are generated based on the performance of student. Since the assessment and feedback is automated, the teachers' workload reduces and provides them with more time to interact with students for their improvement.

The online MCQ based exam system proposed has five major components: the examination preparation system, the examination system, the examination monitor system, data mining component and the auto-grading system[1].

The preparation system: used to manage question storage, assign test ID and schedule the test. The question database is composed of the questions, a set of possible answers, the question types and other metadata, which are indexed by factors, such as topics (units) and difficulty level. The 
database is open to teachers, allowing them to add questions and grade them. Provision of study materials for future examinations is also available.

The examination System: a web-based testing interface for students, which include the following key features: time control, security control and flexibility in the level of questions asked. The students will be allowed for test only within the college network.

The real-time monitoring system: ensures that students not to leave the test window during the test by eliminating access to all other URLs.
Data mining component: used to gather all relevant data and draw conclusion out of it to provide an overall grading to students.

The auto-grading system: designed to provide an instant feedback about the performance of students in the test. This will give the detailed information like the student is good at which units and levels of questions and in which he needs improvement.

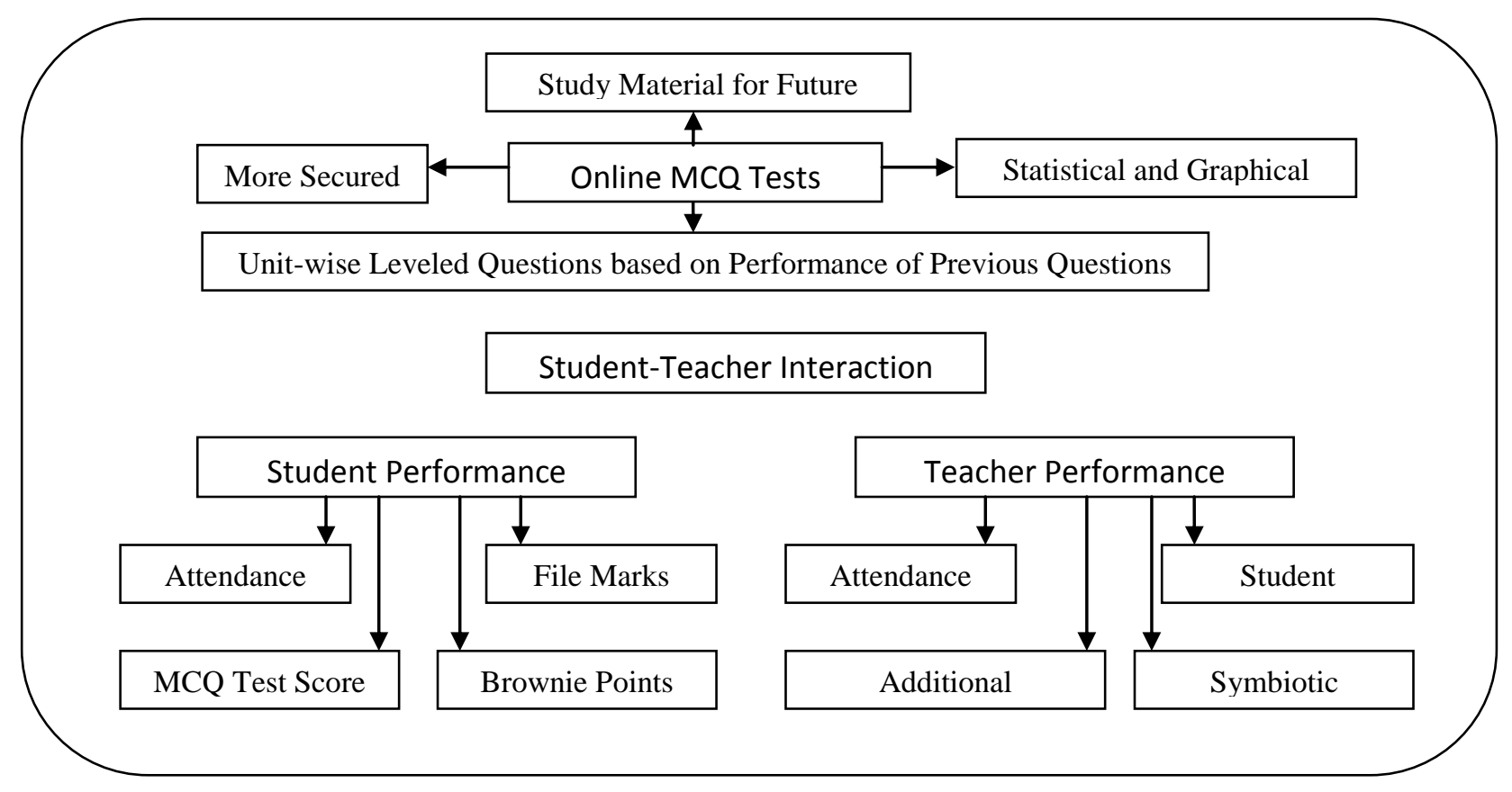

Fig 1: Proposed System Components

The Student performance will be based on the online MCQ based exams, attendance, file marks and some additional brownie points. Brownie points can be for various activities like new algorithm used in practical, delivered an outsourced project, technical paper presentation etc.

The teacher's performance depends on small feedbacks collected from students periodically and attendance. Apart from these, a large contribution is made by information given by the system symbiotically which includes class performance, their contribution to exam question database and some additional work done in the field of interest of the teacher.

The interactive platform proposed in the system enables doubt solving sessions and exchange of notes between teachers and students. Teachers and also review other student's solutions and rank it accordingly.

\section{CONCLUSION:}

Today focus is more on using summative assessment to grade/evaluate students at the end of their learning period for the purpose of certifying achievement, than using formative assessment to help students learn from the test experiences and improve their performance. This compels students to focus on how much marks have they secured rather understanding the content they are studying. The proposed system emphasizes on formative assessment, leading to better development of students. As an added excellence to the system, it also involves interactive sessions between the teachers and students.

\section{ACKNOWLEDGMENT}

It gives us immense pleasure in acknowledging the valuable and co-operative assistance extended to us by the various individuals.

A special thanks to our guide Prof. Supriya Shivanath Kamoji for her guidance throughout the project. We extend our sincere gratitude to the college for providing access to the IEEE and Science Direct papers.

\section{REFERENCES}

[1] Yuan Zhenming, Zhang Liang, Zhan Guohua- "A novel web-based online examination system for computer science education"

[2] Edwina Higgins and Laura Tatham, Manchester Metropolitan University- "Assessing by multiple choice question(MCQ) tests"-http:// www.ukcle.ac.uk/resources /assessment-and-feedback/mcqs

[3] 2010 2nd International Conference on Education Technology and Computer (ICETC) -"Improve student learning using online formative assessment system"

[4] Michael P. Coughlan -"Some observations on mcq tests"

[5] Low Sew Ming - "Reduction of teacher workload in a formative assessment environment through use of online technology"- School of Engineering, Monash University Malaysia 
[6] Necatibey Faculty of Education Electronic Journal of Science and Mathematics Education Vol. 5, Issue 2, December 2011, pp. 18-41 - "Understanding the impact of formative assessment strategies on first year university students' conceptual understanding of chemical concepts" - Mehmet Aydeniz and Aybuke Pabuccu.

[7] Andreas Papasalouros and Konstantinos Kanaris, Konstantinos Kotis - "Automatic generation of multiple choice questions from domain ontologies" - IADIS International Conference e-Learning 2008

[8] Lee Dunn from: Morgan, Chris (1999) Southern Cross University-"Selecting methods of assessment"http://www.brookes.ac.uk/services/ocsld/resources/metho ds.html
[9] A.Salazar, J.Gosalbez, I.Bosch, R.Miralles, L.Vergara, "A case study of knowledge discovery on academic achievement, student desertion and student retention"

[10] Emmanuel N. Ogor, "Student Academic Performance Monitoring and Evaluation Using Data Mining Techniques"

[11] MAO Keji, YU Mingyuan, CHEN Qingzhan,“ Discovering the Most Adaptive Students of One Course by Data Mining"

[12] S. Anupama Kumar and Dr. Vijayalakshmi M.N. "Implication Of Classification Techniques In Predicting Student's Recital". 